\title{
Enhanced stability and antibacterial efficacy of a traditional Chinese medicine-mediated silver nanoparticle delivery system
}

This article was published in the following Dove Press journal:

International Journal of Nanomedicine

26 November 2014

Number of times this article has been viewed

\author{
Wenjie Sun ${ }^{1,2, *}$ \\ Ding $\mathrm{Qu}^{1}{ }^{1, *}$ \\ Yihua $\mathrm{Ma}^{\prime}$ \\ Yan Chen ${ }^{1,2}$ \\ Congyan Liu' \\ Jing Zhou'
}

'Key Laboratory of New Drug

Delivery System of Chinese Materia

Medica, Jiangsu Provincial Academy

of Chinese Medicine, ${ }^{2}$ Department

of Pharmaceutics, School of Pharmacy,

Nanjing University of Chinese

Medicine, Nanjing, Jiangsu, People's

Republic of China

*These authors contributed equally to this work
Correspondence: Yan Chen Key Laboratory of New Drug Delivery System of Chinese Materia Medica, Jiangsu Provincial Academy of Chinese Medicine, 100 Shizi Road, Nanjing, Jiangsu 210028, People's Republic of China

Tel +862585608672

Fax +862585637809

Email ychen202@hotmail.com
Abstract: Silver nanoparticles (AgNPs) are widely used as antibacterial products in various fields. Recent studies have suggested that AgNPs need an appropriate stabilizer to improve their stability. Some antibacterial traditional Chinese medicines (TCMs) contain various reductive components, which can not only stabilize AgNPs but also enhance their antimicrobial activity. In this study, we developed a series of novel AgNPs using a TCM extract as a stabilizer, reducing agent, and antimicrobial agent (TCM-AgNPs). A storage stability investigation of the TCM-AgNPs suggested a significant improvement when compared with bare AgNPs. Further, conjugation of TCMs onto the AgNP surface resulted in stronger antimicrobial potency on antibacterial evaluation using Pseudomonas aeruginosa, Staphylococcus epidermidis, and Staphylococcus aureus with minimum inhibitory concentration 50\% $\left(\mathrm{MIC}_{50}\right)$ ratios (and minimum bactericidal concentration $90 \%\left[\mathrm{MBC}_{90}\right]$ ratios) of AgNPs to respective TCM-AgNPs as assessment indices. Among these, P. cuspidatum Sieb. et-conjugated AgNPs (P.C.-AgNPs) had the advantage of a combination of TCMs and AgNPs and was studied in detail with regard to its synthesis and characterization. The extraction time, reaction temperature, and concentrations of $\mathrm{AgNO}_{3}$ and Polygonum cuspidatum Sieb. et extract were critical factors in the preparation of P.C.-AgNPs. Further, the results of X-ray diffraction and Fourier transform infrared spectroscopy indicated successful preparation of P.C.-AgNPs. In representative studies, P.C.-AgNPs showed a well-defined spherical shape, a homogeneous small particle size $(36.78 \mathrm{~nm})$, a narrow polydispersity index $(0.105)$, and a highly negative zeta potential $(-23.6 \mathrm{mV})$ on transmission electron microscopy and dynamic light scattering. These results indicate that TCM-AgNPs have a potential role as antibacterial agents in the clinic setting.

Keywords: traditional Chinese medicine, extract, silver nanoparticles, stability, antibacterial, Polygonum cuspidatum Sieb. et

\section{Introduction}

Well-designed nanoscale materials have progressively attracted scientific and public concerns about their anti-infective potential. ${ }^{1}$ Among these materials, silver nanoparticles (AgNPs) are of particular interest and have gradually become a primary strategy for antibacterial prophylaxis in wound treatment, sterilization of medical devices, oral health protection, and food sanitation..$^{2-4}$ However, although its antibacterial ability has been adequately proven, the poor stability of nanosilver is a primary limitation to its further clinical application. ${ }^{3}$ Despite the widespread use of AgNPs, there has been little relevant research on their stability and antibacterial properties, with but a few studies in the literature suggesting that the antibacterial activity of AgNPs might be associated with interactions between these particles and 
the bacterial membrane, and that the bacterial response to AgNPs could be easily tunable by altering particle stability, size, and surface charge. ${ }^{5,6}$ Panacek et al have published their interesting results showing that the durability and sustained antibacterial action of AgNPs had a high correlation with their tendency to aggregate and release silver ions. ${ }^{7}$ It appears that stability is a critical factor when AgNPs are to be used for antibacterial treatment. In past decades, the approach most commonly used to address this problem involved modification of citrate on the surface of the nanosilver to obtain acceptable stability. ${ }^{8,9}$ Recently, van der Zande reported that the stability of nanosilver appeared to be greatly improved after modification with polyvinylpyrrolidone in comparison with citrate coating, remaining stable for up to 5 months. ${ }^{10}$ Further, Paredes et al found that certain amino acids could be used as reducing agents in the preparation of nanosilver. ${ }^{4}$ In previous studies, we have prepared AgNPs coated with a natural medicine using Agrimoniae herba extract as the reducing agent and stabilizer, which showed better stability and stronger pharmacological activity than bare AgNPs. ${ }^{11}$

The People's Republic of China has had a great deal of experience in the use of herbal medicines against various bacteria for over 2,000 years. Before the advent of antibiotics, many people with infectious diseases could be saved by numerous antibacterial herbal decoctions. From a modern medicine perspective, these herbal agents contained mainly polyphenols, flavones, and polysaccharides, and the reducing and antimicrobial properties of these compounds are fully understood from previous reports. ${ }^{12-15}$ However, the complexity of component analysis and identification posed a real challenge in the modernization of traditional Chinese medicine (TCM). This was in part because the active ingredients in these decoctions were so complicated that precise quantification of the active substance seemed difficult, resulting in uncertainty with regard to their use in antimicrobial treatment. Since the quick solutions to leaving this predicament have remained elusive within a short time, we should find inspiration from other antimicrobial area for addressing the issues of difficulty of component analysis and identification.

AgNPs and TCM each have their respective advantages and limitations, so combining natural antibacterial medicines and AgNPs seemed reasonable for development of stable and highly effective antibacterial AgNPs. Herein, we report a series of TCM-mediated AgNPs, in which TCM acted not only as an antibacterial agent but also as a stabilizer. This study focused on the rationale of such an approach to enhancing stability and antibacterial efficacy.

\section{Materials and methods Materials}

Polygonum cuspidatum Sieb. et (P.C.), Fagopyrum dibotrys (D. Don), Sanguisorba officinalis L., Agrimonia pilosa Ledeb., Hedyotis diffusa Willd, Rheum palmatum L., and Geranium wilfordii Maxim were provided by Bozhou Pharmaceutical Co Ltd (Bozhou, People's Republic of China). These TCMs were authenticated by Professor Shihui Qian, at the Jiangsu Provincial Academy of Chinese Medicine. Silver nitrate, phosphoric acid, and trisodium citrate were obtained from Sigma-Aldrich (St Louis, MO, USA). Strains of Pseudomonas aeruginosa, Staphylococcus epidermidis, and Staphylococcus aureus were obtained from the Institute of Microbiology, Chinese Academy of Sciences (Beijing, People's Republic of China). Beef extract-peptone medium and phosphate-buffered saline were sourced from Nanjing KeyGEN Biotech Co Ltd (Nanjing, People's Republic of China). Water in this study was provided by a Milli-Q water purification system (Millipore Corporation, Billerica, MA, USA). Other chemicals and reagents were of analytical grade.

\section{Extraction of antibacterial ingredients}

In this experiment, seven antimicrobial TCMs (P.C., F. dibotrys (D. Don), S. officinalis L., A. pilosa Ledeb., H. diffusa Willd, R. palmatum L., and G. wilfordii Maxim) containing various reductive groups were selected to synthesize TCM-AgNPs. ${ }^{16-20}$ These TCM extracts were prepared by using water-coction technology as described in our previous reports. ${ }^{12}$ Briefly, $10 \mathrm{~g}$ of P.C. was cut and boiled for 15 minutes in a $500 \mathrm{~mL}$ round-bottom flask with $100 \mathrm{~mL}$ of deionized water, followed by filtration through filter paper under reduced pressure conditions. Next, the concentration of P.C. extract was adjusted to $0.1 \mathrm{~g}$ crude $\mathrm{drug} / \mathrm{mL}$ and stored at $4^{\circ} \mathrm{C}$ until further use. The other TCM extracts were prepared by similar methods.

\section{Synthesis of TCM-AgNPs}

TCM-AgNPs were synthesized by the AgNP biosynthesis method as previously reported by Niraimathi et al with some modifications. ${ }^{21}$ The specific processes used for preparation of the various TCM-AgNPs were as follows. Briefly, $1 \mathrm{~mL}$ of P.C. extract at a concentration of $0.1 \mathrm{~g}$ crude drug/mL was added to $20 \mathrm{~mL}$ of aqueous solution of 
$\mathrm{AgNO}_{3}(2 \mathrm{mM})$ with vigorously magnetic stirring at room temperature. Subsequently, the acquired P.C.-conjugated AgNPs (P.C.-AgNPs) were separated from the suspension after centrifugation at $13,000 \mathrm{rpm}$ for 15 minutes. Next, the precipitate of P.C.-AgNPs was rinsed three times with deionized water to remove the excess silver ions around and on the surface of the nanoparticles. At the end of collection, a P.C.-AgNP solution was obtained after redispersion in deionized water. The other TCM-NPs were produced by the same method.

\section{Synthesis of bare AgNPs}

Bare AgNPs was synthesized using sodium citrate as a stabilizer according to previous reports. ${ }^{22}$ Briefly, $10 \mathrm{~mL}$ of $1 \%$ sodium citrate solution were added dropwise into $300 \mu \mathrm{g} / \mathrm{mL} \mathrm{AgNO}_{3}$ aqueous solution with intensive stirring at $100^{\circ} \mathrm{C}$. After stirring for 1 hour, the mixture was cooled to room temperature. Finally, AgNPs were obtained at a $\mathrm{pH}$ of 4.0-6.0 using phosphoric acid as a regulator.

\section{Characterization of AgNPs}

\section{Ultraviolet-visible spectroscopy and dynamic light scattering}

Ultraviolet-visible absorption spectra for bare AgNPs and TCM-AgNPs were recorded to observe the surface plasmon resonance (SPR) absorption band for confirming the formation of AgNPs at a wavelength of 300-800 nm using a UV-1800 PC ultraviolet-visible spectrophotometer (AoXi Technology Instruments, Shanghai, People's Republic of China). In addition, the particle size and zeta potential of various AgNPs were measured using a Zetasizer (Nano ZS: Malvern, UK) after 20-fold dilution.

\section{Morphology}

Transmission electron microscopy (TEM) was performed to observe the morphology of the optimized P.C.-AgNP solution by dropping $15 \mu \mathrm{L}$ of nanoparticles onto a holey carbon-coated copper grid and drying prior to measurement (Tecnai 12, Philips, Amsterdam, the Netherlands). The average particle size of the P.C.-AgNPs was calculated by counting and analyzing the size of 400 nanoparticles in ten fields of vision selected randomly from TEM images.

\section{X-ray diffraction measurements}

$\mathrm{X}$-ray diffraction spectra were measured to characterize the crystalline structure of the optimal P.C.-AgNPs by X-ray diffraction (D8 Advance; Bruker Optik, Ettlingen, Germany) operated at $40 \mathrm{kV} / 40 \mathrm{~mA}$. The pattern was recorded with $\mathrm{CuK} \alpha$ radiation $(\lambda=1.5406 \AA)$ in the range of $10^{\circ}-80^{\circ}$ at a scanning rate of $2^{\circ}$ per minute.

\section{Fourier transform infrared spectroscopy}

Fourier transform infrared (FTIR, Nicolet IS10; Thermo Fisher Scientific, Waltham, MA, USA) spectra were recorded over a range of 4,000-500 $\mathrm{cm}^{-1}$ using $2^{\circ}$ per $\mathrm{cm}$ resolution to confirm conjugation of the P.C. extract with the surface of the nanoparticles.

\section{Stability studies}

In order to identify the advantages of TCMs over sodium citrate as a stabilizer for AgNPs, the storage stability of various TCM-AgNPs and bare AgNPs was investigated by recording their size changes at $0,7,14,21$, and 30 days using dynamic light scattering. Using the ultraviolet-visible method mentioned above, absorption spectra for P.C.-AgNPs stored for 0 and 2 months were recorded in the range of 300-800 nm, respectively.

\section{Antibacterial assays}

In this study, $P$. aeruginosa, $S$. epidermidis, and $S$. aureus, commonly encountered pathogenic bacteria in the clinical setting, were used to evaluate the antibacterial activity of the TCM extracts, bare AgNPs, and TCM-AgNPs via a conventional method. ${ }^{23-25}$ Briefly, P. aeruginosa, S. epidermidis, and $S$. aureus were cultivated in beef extract-peptone medium at $37^{\circ} \mathrm{C}$ for 12 hours prior to the experiment. The cultures were diluted 1,000 times with phosphate-buffered saline to obtain bacterial suspensions containing approximately $10^{6}$ colony forming units $/ \mathrm{mL}$. Next, $50 \mu \mathrm{L}$ gradient concentrations of TCM extracts $\left(0.5 \times 10^{3}, 2 \times 10^{3}, 5 \times 10^{3}, 1 \times 10^{4}, 2 \times 10^{4}, 5 \times 10^{4}\right.$, and $\left.1 \times 10^{5} \mu \mathrm{g} / \mathrm{mL}\right)$, bare AgNPs, and TCM-AgNPs $(0.5,1,2$, $5,10,20,50$, and $100 \mu \mathrm{g} / \mathrm{mL}$ ) were mixed thoroughly with $50 \mu \mathrm{L}$ of culture medium in 96-well plates. The plates were then sealed with sealing film and incubated for 12 hours at $37^{\circ} \mathrm{C}$. Optical density was determined at $595 \mathrm{~nm}$ for bacterial cell growth using a microplate reader (Thermo Fisher Scientific) at $37^{\circ} \mathrm{C}$.

The minimum inhibitory concentration (MIC) and minimum bactericidal concentration (MBC) were defined as the lowest concentrations of drugs that produce inhibition and sterilization of bacterial growth, respectively. $\mathrm{MIC}_{50}$ and $\mathrm{MBC}_{90}$ values corresponded to doses that inhibit 50\% and sterilize $90 \%$ of bacterial growth, respectively. 


\section{Optimization of P.C.-AgNP synthesis}

To evaluate their relative influence on the synthesis and yield of P.C.-AgNPs, different synthetic conditions, including extraction time, reaction temperature, the concentration of $\mathrm{AgNO}_{3}$, and amount of P.C. extract, were investigated and optimized comprehensively. As in our previous papers, ${ }^{11,26}$ the extraction time of $P$.C. was predetermined at 2, 5, 15, 30, and 60 minutes to evaluate the effect on the yield of P.C.AgNPs. Likewise, the reaction temperature was preset at $4^{\circ} \mathrm{C}$, $25^{\circ} \mathrm{C}, 40^{\circ} \mathrm{C}, 50^{\circ} \mathrm{C}$, and $60^{\circ} \mathrm{C}$ to explore whether there was a time-dependent relationship in the synthesis of P.C.-AgNPs. Further, various concentration gradients of $\mathrm{AgNO}_{3}$ set at $0.5 \mathrm{mM}, 1.0 \mathrm{mM}, 2.0 \mathrm{mM}, 3.0 \mathrm{mM}$, and $4.0 \mathrm{mM}$ were used to observe the influence of concentration on the synthesis of P.C.-AgNPs. Finally, 0.25, 0.5, 1, 2.5, 5, and $7.5 \mathrm{~g}$ of crude drug at a concentration of $0.1 \mathrm{~g} / \mathrm{mL}$ were mixed with $100 \mathrm{~mL}$ of $2 \mathrm{mM}$ aqueous $\mathrm{AgNO}_{3}$ solution to evaluate the effect of amount of P.C. on the preparation of P.C.-AgNPs. In this experiment, ultraviolet-visible spectral analysis was done for signal factor optimization and to evaluate the rate of synthesis of P.C.-AgNPs prepared under different reaction conditions at a wavelength of 300-800 nm.

\section{Statistical analysis}

All experiments were carried out in triplicate unless otherwise specified. All statistical analyses were performed using GraphPad Prism 6 and the data were expressed as the mean \pm standard deviation. Differences between groups were evaluated using the Student's $t$-test. A $P$-value $<0.01$ was considered to be statistically significant.

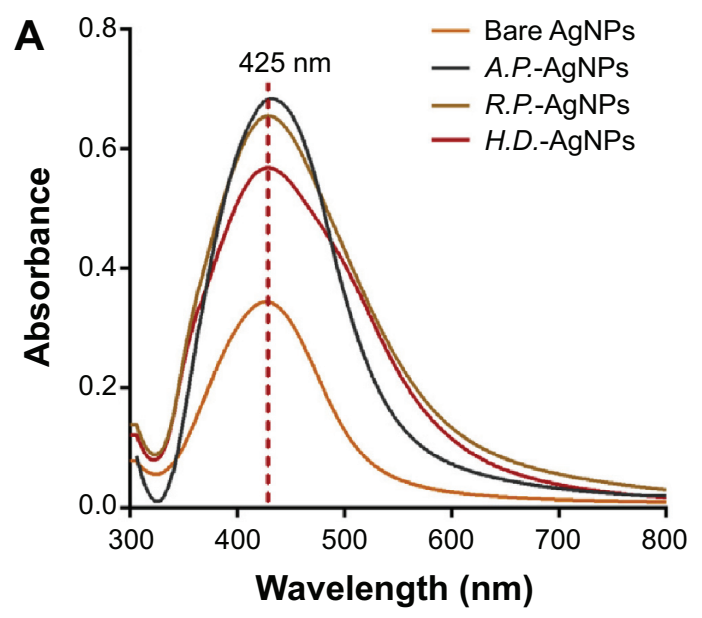

\section{Results and discussion}

\section{Preparation and characterization of TCM-AgNPs}

Prior to estimating their antibacterial activity, the synthesis and physicochemical characterization of bare AgNPs and TCMAgNPs were studied according to the conventional method with some modifications. Some studies have indicated previously that free AgNP electrons gave an SPR absorption band due to the combined vibration of electrons in resonance with light waves. ${ }^{27}$ In view of this, the synthesized AgNPs were primarily characterized by ultraviolet-visible spectroscopy, which has proved to be a very useful technique for evaluating the yield as well as the characteristics of nanosilver. ${ }^{28}$ In the ultraviolet-visible spectrum, strong SPR peaks (Figure 1) were observed for bare AgNPs and the various TCM-AgNPs, suggesting successful synthesis of AgNPs. In addition, as shown in Figure 1A and B, strong SPR peaks for bare AgNPs, A.P.-AgNPs, R.P.-AgNPs, and H.D.-AgNPs (A. pilosa Ledeb.-coated AgNPs, R. palmatum L.-coated AgNPs, and H. diffusa Willd-coated AgNPs, respectively) were found at about $425 \mathrm{~nm}$, while that of other TCM-AgNPs were located at about $445 \mathrm{~nm}$, suggesting that AgNPs with different coatings displayed different maximum absorption peaks.

Further characterization was carried out using a dynamic light scattering analyzer. As shown in Table 1, both TCMAgNPs and bare AgNPs had a small particle size of around $50 \mathrm{~nm}$. Among the TCM-AgNPs that we prepared, P.C.AgNPs had the narrowest polydispersity index and a highly negative zeta potential when compared with bare AgNPs, suggesting an acceptable preparation technology and a

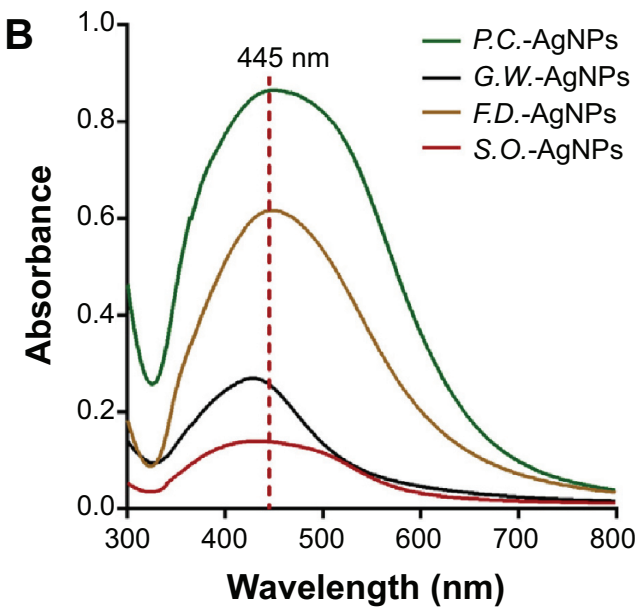

Figure I Ultraviolet-visible absorption spectra of AgNPs and various TCM-AgNPs within a range of 300-800 nm.

Notes: Among these AgNPs, (A) the maximum absorption peaks of bare AgNPs and some TCM-AgNPs are located at about $425 \mathrm{~nm}$, (B) while those of other TCM-AgNPs are observed at about $445 \mathrm{~nm}$.

Abbreviations: AgNPs, silver nanoparticles; A.P.-AgNPs, Agrimonia pilosa Ledeb.-coated AgNPs; R.P.-AgNPs, Rheum palmatum L.-coated AgNPs; H.D.-AgNPs, Hedyotis diffusa Willd-coated AgNPs; P.C.-AgNPs, Polygonum cuspidatum Sieb. et-coated AgNPs; G.W.-AgNPs, Geranium wilfordii Maxim-coated AgNPs; F.D.-AgNPs, Fagopyrum dibotrys (D. Don)-coated AgNPs; S.O.-AgNPs, Sanguisorba officinalis L.-coated AgNPs; TCM, traditional Chinese medicine. 
Table I Physicochemical characterization of bare AgNPs and TCM-AgNPs

\begin{tabular}{llll}
\hline Formulations & Particle size $(\mathbf{n m})$ & PDI & Zeta potential (mV) \\
\hline Bare AgNPs & $36.19 \pm 4.7$ & $0.249 \pm 0.039$ & $-12.1 \pm 1.6$ \\
P.C.-AgNPs & $36.78 \pm 3.1$ & $0.105 \pm 0.017$ & $-23.6 \pm 2.1$ \\
A.P.-AgNPs & $48.21 \pm 1.8$ & $0.168 \pm 0.021$ & $-20.9 \pm 1.7$ \\
R.P.-AgNPs & $38.15 \pm 3.6$ & $0.127 \pm 0.009$ & $-20.6 \pm 2.5$ \\
H.D.-AgNPs & $39.97 \pm 2.3$ & $0.181 \pm 0.026$ & $-21.3 \pm 1.9$ \\
F.D.-AgNPs & $45.49 \pm 2.9$ & $0.188 \pm 0.019$ & $-19.7 \pm 3.2$ \\
S.O.-AgNPs & $53.71 \pm 5.1$ & $0.114 \pm 0.006$ & $-17.2 \pm 2.2$ \\
G.W.-AgNPs & $50.97 \pm 4.7$ & $0.149 \pm 0.016$ & $-21.8 \pm 2.4$ \\
\hline
\end{tabular}

Abbreviations: AgNPs, silver nanoparticles; PDI, polydispersity index; TCM-AgNPs, traditional Chinese medicine-coated AgNPs; P.C.-AgNPs, Polygonum cuspidatum Sieb. et-coated Ag nanoparticles; A.P.-AgNPs, Agrimonia pilosa Ledeb.-coated AgNPs; R.P.-AgNPs, Rheum palmatum L.-coated AgNPs; H.D.-AgNPs, Hedyotis diffusa Willd-coated AgNPs; F.D.-AgNPs, Fagopyrum dibotrys (D. Don)-coated AgNPs; S.O.-AgNPs, Sanguisorba officinalis L.-coated AgNPs; G.W.-AgNPs, Geranium wilfordii Maxim-coated AgNPs.

potentially stable system. It was clear that incorporation of TCM extract into the surface of AgNPs significantly changed the particle size, polydispersity index, and zeta potential, indicating an advantage of improved stability when compared with bare nanosilver.

\section{Stability evaluation of TCM-AgNPs}

In order to confirm the enhanced stability of AgNPs, the aggregation behavior of the various AgNPs was investigated by observation of size changes at predetermined time points and room temperature (Figure 2A). Based on the results obtained, the size of the bare AgNPs gradually increased from $36 \mathrm{~nm}$ to $375 \mathrm{~nm}$ in the absence of an effective stabilizing agent, indicating aggregation after 14 days. In contrast, little alteration in the average size of the TCM-AgNPs was observed after 30 days of storage, suggesting improved stability of the AgNPs after modification with TCM extract. The advantage of using TCM extracts as a protective agent was confirmed again from the perspective of storage stability. Based on the above physicochemical characterization, we speculated that a highly negative potential and a small and well distributed size could afford P.C.-AgNPs excellent stability. As shown in Figure 2B, no notable change was found in the ultraviolet absorption of P.C.-AgNPs, indicating that the AgNPs remained stable for up to 2 months after coating with the P.C. extract.

\section{Analysis of antibacterial properties}

According to previous studies, an appropriate coating (reductive/stabilizing agent in this study) can alter the release profile of silver ions, and this is a critical factor in the antibacterial activity of AgNPs. ${ }^{10}$ With the aim of confirming
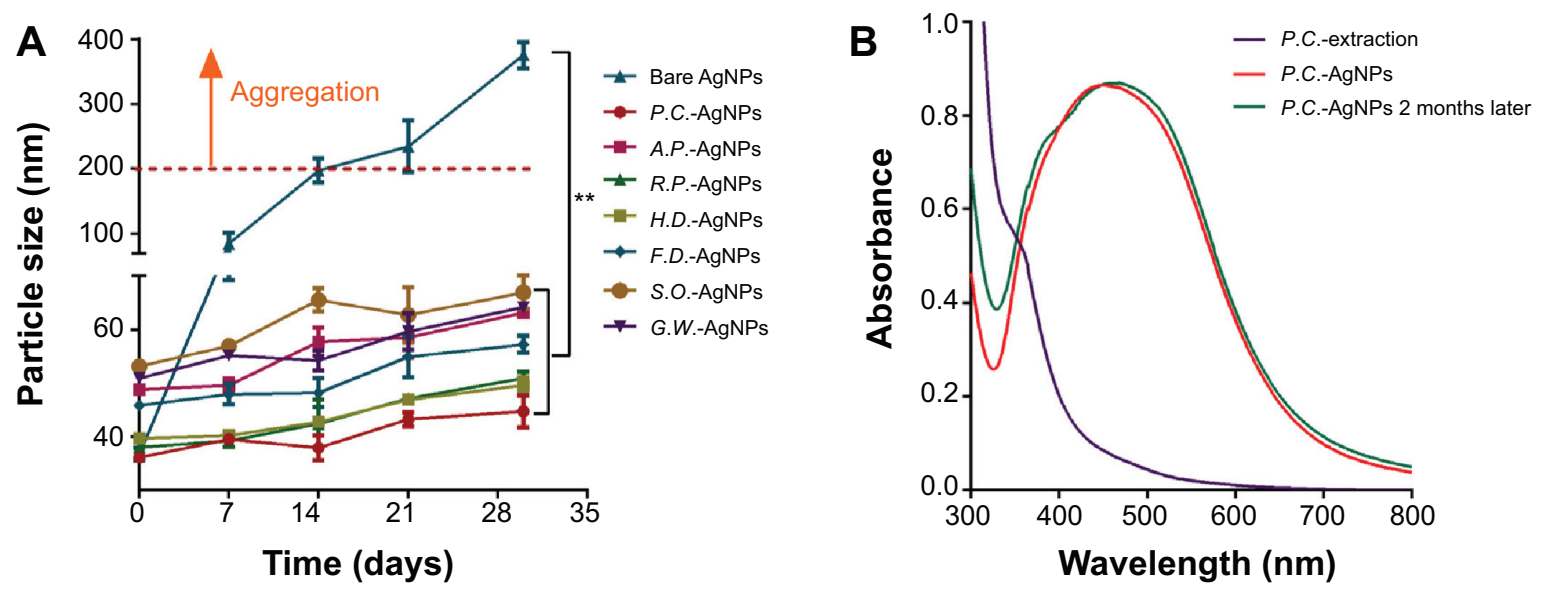

Figure 2 Evaluation of long-term storage stability. (A) Particle size alteration of bare AgNPs and TCM-AgNPs after storage for 0, 7, I4, 2I, and 30 days ( $\mathrm{n}=3$ ). (B) Stability studies of P.C.-AgNPs stored for 2 months at room temperature observed for ultraviolet-visible absorption.

Notes: $* * P<0.01$ versus TCM-AgNPs. Data are shown as the mean \pm standard deviation. The dotted line in $\mathbf{A}$ indicates that a particle size larger than $200 \mathrm{~nm}$ was speculated to be aggregation.

Abbreviations: AgNPs, silver nanoparticles; A.P.-AgNPs, Agrimonia pilosa Ledeb.-coated AgNPs; R.P.-AgNPs, Rheum palmatum L.-coated AgNPs; H.D.-AgNPs, Hedyotis diffusa Willd-coated AgNPs; P.C.-AgNPs, Polygonum cuspidatum Sieb. et-coated AgNPs; G.W.-AgNPs, Geranium wilfordii Maxim-coated AgNPs; F.D.-AgNPs, Fagopyrum dibotrys (D. Don)-coated AgNPs; S.O.-AgNPs, Sanguisorba officinalis L.-coated AgNPs; TCM, traditional Chinese medicine. 
the advantages of TCMs as a coating for AgNPs in terms of antimicrobial activity, conventional bacterial inhibition assays were performed against $P$. aeruginosa, $S$. epidermidis, and $S$. aureus in vitro. In this experiment, we focused on identification of exactly which component contributed most to the antibacterial effects. Therefore, $\mathrm{MIC}_{50}$ ratios of TCM extract to TCM-AgNPs and $\mathrm{MIC}_{50}$ ratios of bare AgNPs to
TCM-AgNPs were used to evaluate the contribution of AgNPs and TCM extracts to the antimicrobial activity, respectively, as well as the corresponding $\mathrm{MBC}_{90}$ ratios. As shown in Figure 3A, both TCM extract and AgNPs significantly promoted the antibacterial potency of the various TCM-AgNPs. Taking P.C.-AgNPs as an example, the antibacterial effect on $P$. aeruginosa was enhanced by about 30,000 times

\section{A Pseudomonas aeruginosa}
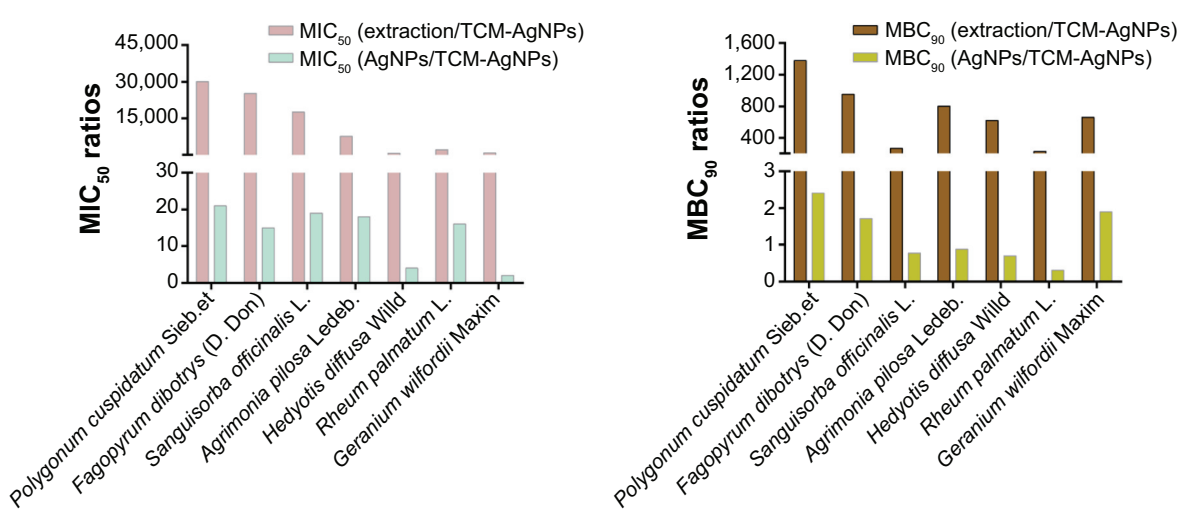

B Staphylococcus epidermidis
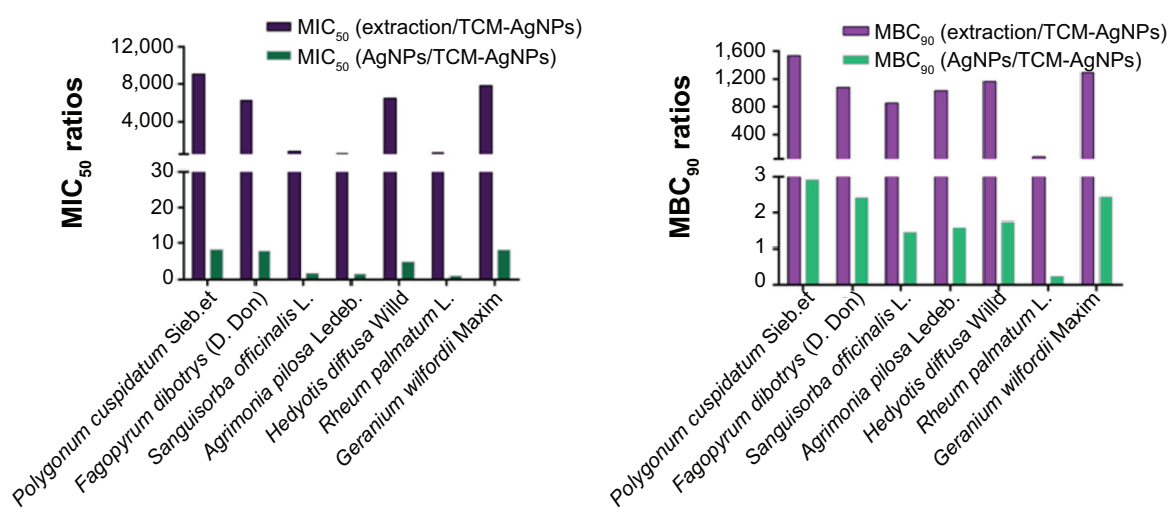

C Staphylococcus aureus

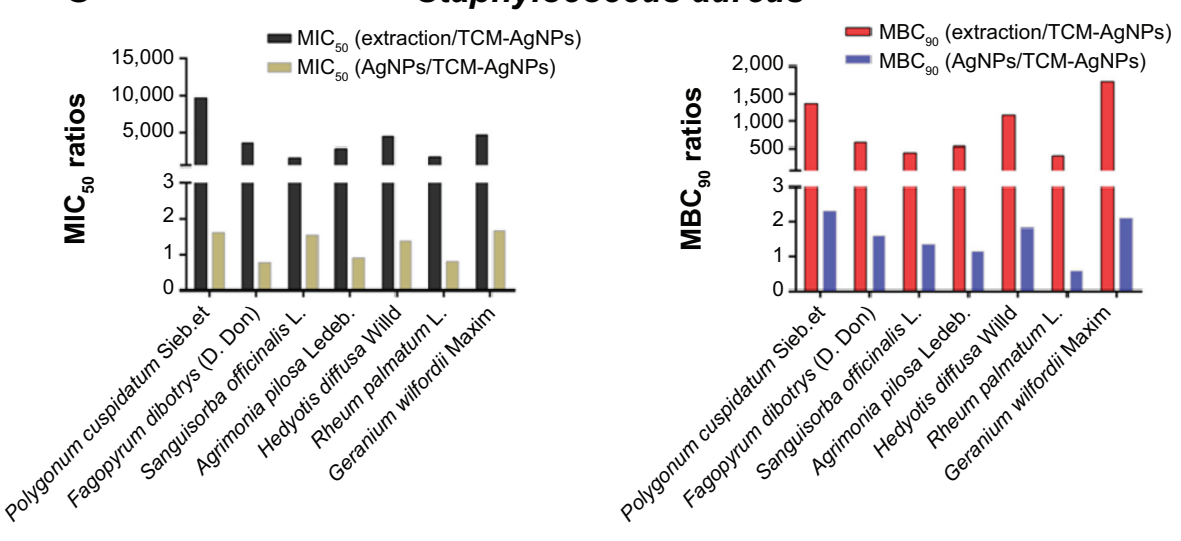

Figure 3 Contribution factor analysis to antibacterial effect. Antimicrobial influence of AgNPs and TCM extractions against (A) Pseudomonas aeruginosa, (B) Staphylococcus epidermidis, and (C) Staphylococcus aureus.

Notes: $\mathrm{MIC}_{50}$ ratios of TCM extraction (or AgNPs) to TCM-AgNPs represented the contribution of bare AgNPs or TCM extractions to bacterial inhibition. MBC 90 ratios of TCM extracts (or AgNPs) to TCM-AgNPs represent the contribution of bare AgNPs or TCM extracts to antibacterial ability.

Abbreviations: TCM, traditional Chinese medicine; $\mathrm{AgNPs}$, silver nanoparticles; $\mathrm{MIC}_{50}$, minimum inhibitory concentration that inhibits $50 \%$ of bacterial growth; $\mathrm{MBC}{ }_{90}$, minimum bactericidal concentration that sterilizes $90 \%$ of bacterial growth. 
when compared with the P.C. extract, suggesting that the AgNPs were a predominant contributor to the antibacterial activity. Meanwhile, the $\mathrm{MIC}_{50}$ for P.C.-AgNPs was 20-fold higher than that for bare AgNPs, indicating a synergistic effect between TCM extract and AgNPs on antibacterial activity. Likewise, $\mathrm{MBC}_{90}$ ratios of TCM extract to TCMAgNPs and $\mathrm{MBC}_{90}$ ratios of bare AgNPs to TCM-AgNPs showed a similar tendency. As shown Figure 3B and C, introduction of P.C. and G. Wilfordii Maxim as coatings had the most significant effect on antibacterial activity. It is noteworthy that the specific contributions of various factors to pharmacological function were influenced by bacterial strains, TCM species, and selection of indices. In view of their most prominent antimicrobial performance, P.C.-AgNPs were screened to perform further synthesis optimization and physicochemical characterization.

In this study, the reductive natural ingredients were coated with the AgNPs quantitatively, and the inactive portion of the crude drug was removed during preparation. Therefore, conjugation of TCM extract and AgNPs not only enhanced the antibacterial activity, but also reduced the uncertainty of TCM in antibacterial therapy, exhibiting the merit of this strategy again in the perspective of treatment reliability.
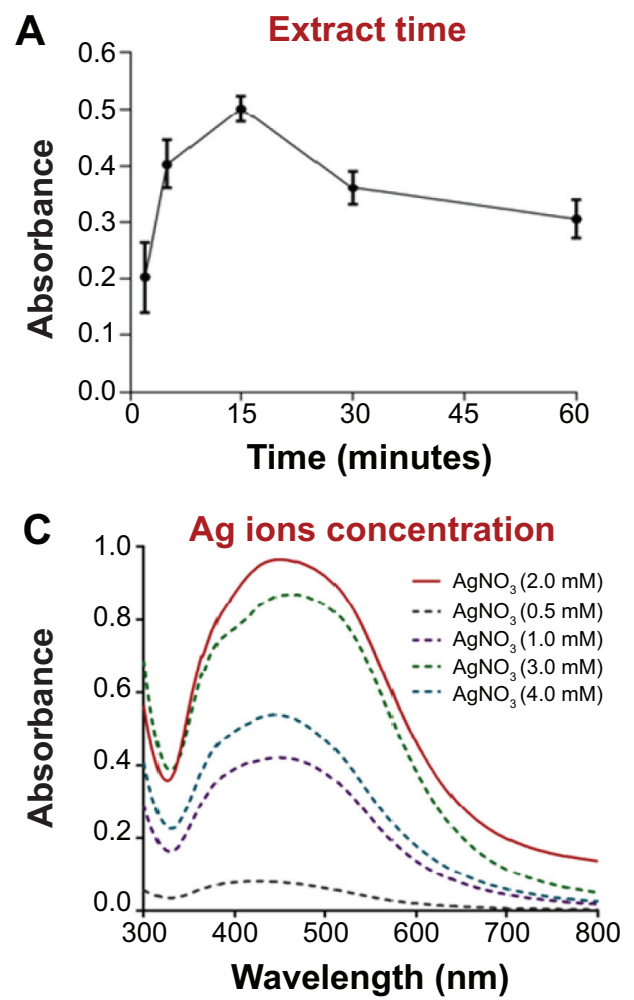

\section{Optimization of P.C.-AgNP synthesis} Effect of extraction time

According to the potential mechanism of formation of P.C.-AgNPs, P.C. extract containing reductive groups interacted with silver ions via a redox reaction. Therefore, the amount of reductive components in the $P$.C. extract was of great importance in the synthesis of P.C.-AgNPs and was directly related to the extraction time. In this experiment, the ultraviolet-visible absorption of P.C.-AgNPs at the maximum wavelength $(445 \mathrm{~nm})$ was recorded at different extraction times to evaluate the conjugation efficiency (Figure 4A). The maximum amount of P.C. conjugated with AgNPs was found after an extraction time of 15 minutes. Interestingly, the longer extraction time of $P$.C. resulted in a comparatively low yield of P.C.-AgNPs, mainly because the reductive component was destroyed after being boiled for longer than 15 minutes. This suggests that extraction time plays an important role in bioreduction.

\section{Effect of reaction conditions}

Our previous studies found that both reaction temperature and time significantly influenced the yield of TCM-AgNPs. In the present study, temperatures ranging from $4^{\circ} \mathrm{C}$ to $60^{\circ} \mathrm{C}$
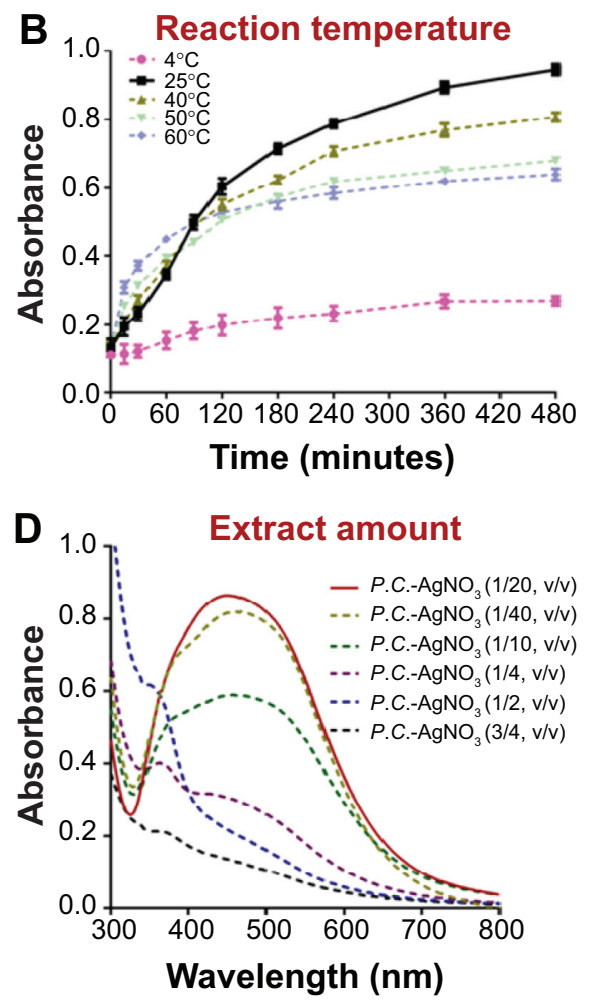

Figure 4 Single factor optimization of P.C.-AgNP synthesis. (A) Influence of extraction time on yield of P.C.-AgNPs, (B) impact of reaction temperature and time on preparation of TCM-AgNPs, and effect of (C) $\mathrm{AgNO}_{3}$ concentration, and (D) amount of P.C. extract on the synthesis of P.C.-AgNPs.

Notes: Data are shown as the mean \pm standard deviation. The solid lines represent the optimum reaction conditions, and the dotted lines represent assayed reaction conditions.

Abbreviations: AgNPs, silver nanoparticles; P.C.-AgNPs, Polygonum cuspidatum Sieb. et-coated AgNPs; TCM, traditional Chinese medicine. 
were used to investigate the influence of temperature on the yield over time by observing the peak wavelength of P.C.AgNPs. As shown in Figure 4B, an extension of reaction time was accompanied by an obvious improvement in the yield of P.C.-AgNPs. Importantly, temperatures below or above $25^{\circ} \mathrm{C}$ could not improve the maximum synthesis rate of $P$.C.AgNPs. This suggests that a continuous temperature higher than $25^{\circ} \mathrm{C}$ might inactivate the reductive component of the extract and is not conducive to a reducing reaction.

\section{Effect of silver ion concentration}

Bioreduction of silver ions mediated by P.C. extract was also monitored by recording the absorption spectra at different $\mathrm{AgNO}_{3}$ concentrations. As shown in Figure 4C, absorbance at $445 \mathrm{~nm}$ increased as the concentration increased from $0.5 \mathrm{mM}$ to $2.0 \mathrm{mM}$. However, the maximum absorption wavelength was shifted when the concentration of $\mathrm{AgNO}_{3}$ was higher than $2.0 \mathrm{mM}$, suggesting that the maximum yield of P.C.-AgNPs was obtained at a silver ion concentration of $2.0 \mathrm{mM}$.

\section{Effect of amount of P.C. extract}

Optimization of the amount of P.C. extract was carried out by varying the ratio of extract solution to silver nitrate (1:40, $1: 20,1: 10,1: 4,1: 2$, and $3: 4, \mathrm{v} / \mathrm{v})$. Various ultraviolet-visible spectra for P.C.-AgNPs (Figure 4D) indicated that when the ratio of $P$.C. extract to $2 \mathrm{mM}$ of $\mathrm{AgNO}_{3}$ was predetermined at 1:20 (v/v), the optimal yield of P.C.-AgNPs was achieved. In addition, the prepared P.C.-AgNP solution had a dark-brown appearance, which might have contributed to the occurrence of SPR effect. This further confirmed successful preparation of P.C.-AgNPs through a visual observation method. ${ }^{29-31}$

\section{Morphology of P.C.-AgNPs}

TEM was used to investigate the microstructure of P.C.AgNPs and to evaluate the relevant preparation technology. As shown in Figure 5A, P.C.-AgNPs had a spherical morphology with a homogeneous dispersion. By statistically measuring the size of 400 nanoparticles from ten randomly selected visual fields in the TEM images, we found that the size was around $12 \mathrm{~nm}$ (Figure 5B). Compared with the TEM images, the size observed by dynamic light scattering results was significantly decreased, mainly as a result of the hydrodynamic size in an aqueous system. ${ }^{32}$ Taken together with the dynamic light scattering results, these findings indicate a superior and highly efficient preparation technology for P.C.-AgNPs and a stable system contributed to by a highly negative zeta potential.

\section{Powder X-ray diffraction analysis}

The formation of AgNPs was further confirmed by the X-ray diffraction pattern. Figure 6A (original Figure is provided in the Figure S1) shows four distinct diffraction peaks at $38.35^{\circ}, 44.56^{\circ}, 64.74^{\circ}$, and $77.62^{\circ}$ which were attributed to the (111), (200), (220), and (311) crystallographic planes of the face-centered cubic silver crystals, respectively. ${ }^{33}$ Further, these diffraction peaks in the X-ray diffractogram were consistent with the database of the Joint Committee on Powder Diffraction Standards file No. 04-0783. The average
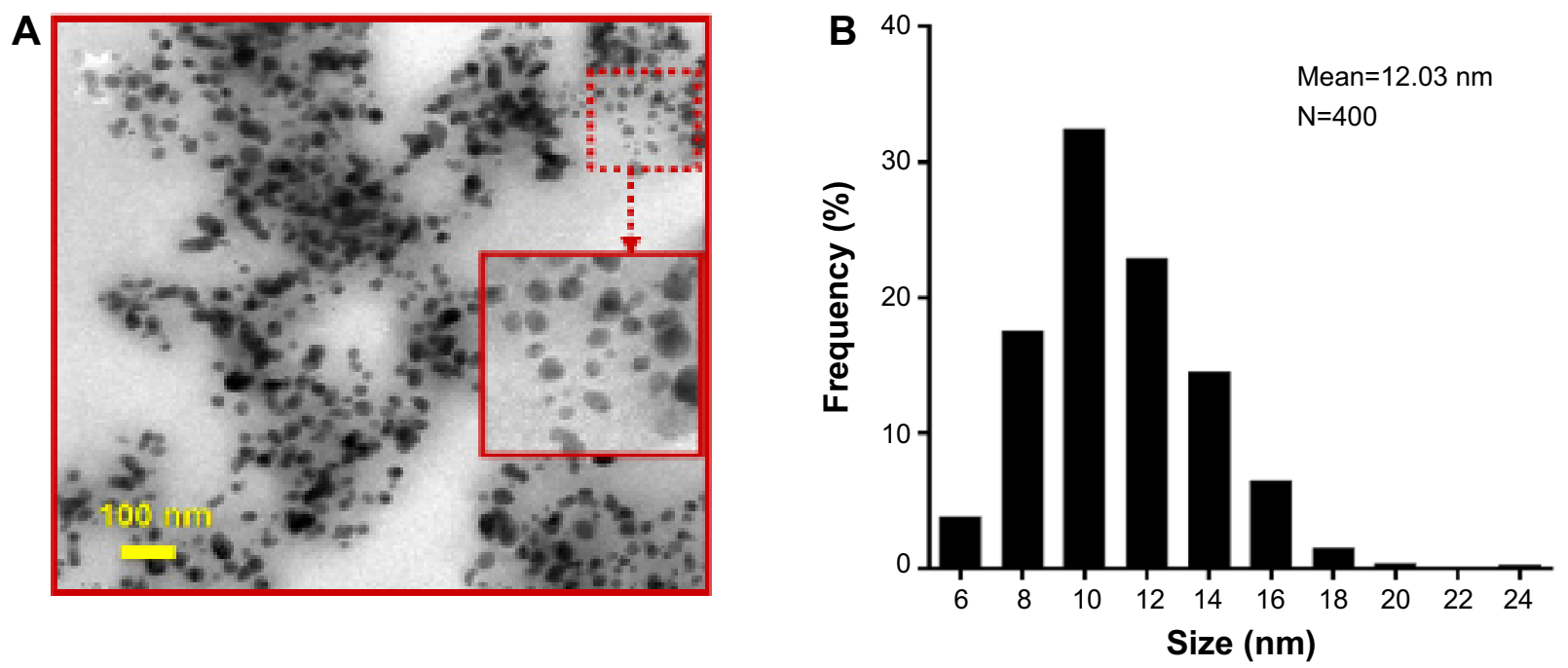

Figure 5 Morphologic studies of P.C.-AgNPs.

Notes: (A) TEM image and (B) statistical measurement of P.C.-AgNP size distribution from ten randomly selected visual fields on the TEM image. Abbreviations: P.C.-AgNPs, Polygonum cuspidatum Sieb. et-coated silver nanoparticles; TEM, transmission electron microscopy. 
A

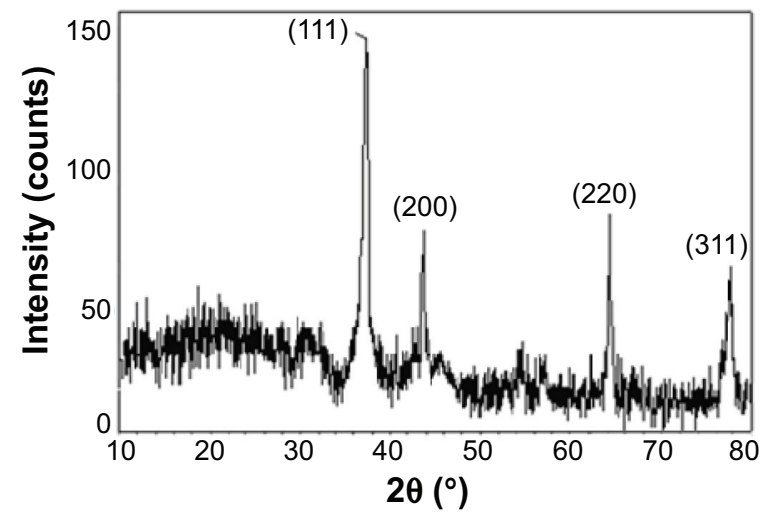

B

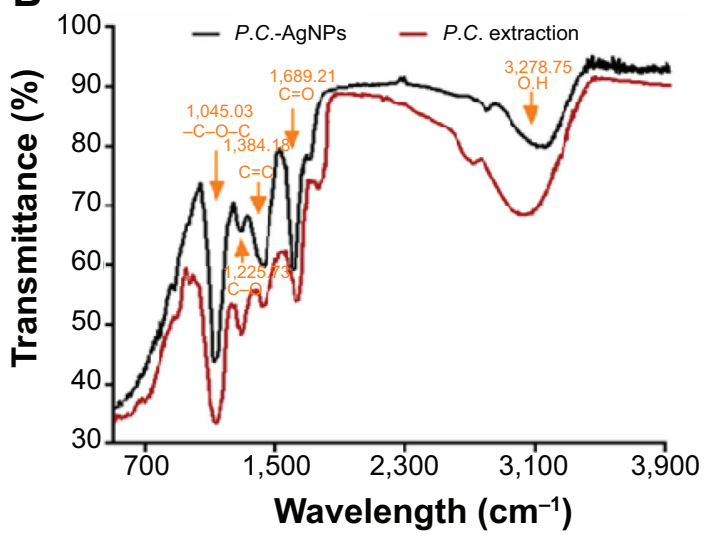

Figure 6 Structural characterization of P.C.-AgNPs.

Notes: (A) X-ray diffraction patterns of P.C.-AgNPs and (B) Fourier transform infrared absorption spectra of P.C. extract and P.C.-AgNPs freeze-dried power. Abbreviation: P.C.-AgNPs, Polygonum cuspidatum Sieb. et-coated silver nanoparticles.

size was calculated according to the Debye-Scherrer equation determining the width of the Bragg's reflection:

$$
\mathrm{D}=\mathrm{K} \lambda / \beta \cos \theta
$$

where $\mathrm{D}$ is the mean crystal size of the nanoparticles, $\mathrm{K}$ represents the Scherrer constant with a value of $0.89, \lambda$ represents the X-ray wavelength ( $1.5406 \AA), \beta$ is the full width at half the maximum intensity in radians, and $\theta$ represents the Bragg angle. According to Debye-Scherrer's equation, the average crystallite size of P.C.-AgNPs was calculated to be about $12.24 \mathrm{~nm}$ (Table 2), which was in line with the result obtained from the TEM images.

\section{FTIR spectroscopy analysis}

The FTIR absorption spectra for the P.C. extract and the P.C.-AgNP freeze-dried powder shown in Figure $6 \mathrm{~B}$ (original Figures are provided in the Figure $\mathrm{S} 2$ and Figure $\mathrm{S} 3$ ) confirm the successful conjugation of P.C. and AgNPs. Compared with the P.C. extract, the characteristic peak of P.C.-AgNPs at $3,278.75 \mathrm{~cm}^{-1}$, which was assigned to the bending vibration of the hydroxyl group, was obviously weak according to the FTIR spectra, suggesting that consumption of hydroxyl

Table 2 X-ray diffraction measurements of P.C.-AgNPs for evaluating average crystallite size

\begin{tabular}{lllll}
\hline (hkl) & $\mathbf{2 \theta}$ (deg) & $\boldsymbol{\theta}$ (deg) & $\boldsymbol{\beta}$ (rad) & $\mathbf{D}(\mathbf{n m})$ \\
\hline$(\mathrm{III})$ & 38.35 & 19.18 & 0.0131 & 11.12 \\
$(200)$ & 44.56 & 22.28 & 0.0129 & 11.47 \\
$(220)$ & 64.74 & 32.37 & 0.0125 & 12.96 \\
$(3 \mathrm{II})$ & 77.62 & $38.8 \mathrm{I}$ & 0.0131 & 13.42 \\
\hline
\end{tabular}

Notes: $D$ represents the mean crystal size of P.C.-AgNPs, $\beta$ represents the width of the XRD peak at half-height, and $\theta$ represents the Bragg angle.

Abbreviations:P.C.-AgNPs, Polygonumcuspidatum Sieb.et-coatedsilvernanoparticles; $X R D, X$-ray diffraction. groups was responsible for the reduction of the silver ions. ${ }^{34-36}$ In addition, the FTIR spectra for P.C.-AgNPs showed strong absorption bands at 1,689.21, 1,384.18, 1,225.73, and $1,045.03 \mathrm{~cm}^{-1}$, which were specifically associated with $\mathrm{C}=\mathrm{O}$ stretching aliphatic amine, $\mathrm{C}=\mathrm{C}$ aromatic, $\mathrm{C}-\mathrm{O}$ stretching acidic, and $-\mathrm{C}-\mathrm{O}-\mathrm{C}$ stretching vibration, respectively. These vibrational bands corresponding to the bonds were derived from water-soluble extracts such as flavonoids and polyphenols present in the P.C. extract. ${ }^{37-40}$ This suggests that the reductive components mentioned above were coated on the surface of the nanoparticles and hence may have inhibited further aggregation in an aqueous environment.

\section{Conclusion}

In summary, seven TCM-AgNPs were synthesized using a simple and highly effective preparation technology with potential for use in industrial production. Meanwhile, various TCM extractions as reductive agents and stabilizer enhanced the storage stability of TCM-AgNPs significantly. Importantly, these TCM-AgNPs were highly effective in inhibiting growth of $P$. aeruginosa, $S$. epidermidis, and $S$. aureus. Further, the different TCMAgNPs had different activity against the three bacterial strains used in this study. Typically, the conjugation of P.C. extract and AgNPs was helpful to enhancement on inhibition of common bacteria. In conclusion, the results of this study suggest that TCM-AgNPs have the potential to be a promising drug delivery system for antibacterial therapy.

\section{Acknowledgments}

This work was financially supported by the Jiangsu Provincial Chinese Medicine Leading Talent Project (LJ200913) and the National Natural Science Foundation (81373979). 


\section{Disclosure}

The authors report no conflicts of interest in this work.

\section{References}

1. Zhao L, Wang H, Huo K, et al. Antibacterial nano-structured titania coating incorporated with silver nanoparticles. Biomaterials. 2011; 32(24):5706-5716.

2. Prabhu S, Poulose EK. Silver nanoparticles: mechanism of antimicrobial action, synthesis, medical applications, and toxicity effects. Int Nano Lett. 2012;32(2):1-10.

3. Alarcon EI, Udekwu K, Skog M, et al. The biocompatibility and antibacterial properties of collagen-stabilized, photochemically prepared silver nanoparticles. Biomaterials. 2012;33(19):4947-4956.

4. Paredes D, Ortiz C, Torres R. Synthesis, characterization, and evaluation of antibacterial effect of Ag nanoparticles against Escherichia coli O157: h7 and methicillin-resistant Staphylococcus aureus (MRSA). Int J Nanomedicine. 2014;9:1717-1729.

5. Nel A, Xia T, Mädler L, Li N. Toxic potential of materials at the nanolevel. Science. 2006;311(5761):622-627.

6. dos Santos CA, Jozala AF, Pessoa A Jr, Seckler MM. Antimicrobial effectiveness of silver nanoparticles co-stabilized by the bioactive copolymer pluronic F68. J Nanobiotechnology. 2012;10:43.

7. Panacek A, Kvítek L, Prucek R, et al. Silver colloid nanoparticles: synthesis, characterization, and their antibacterial activity. J Phys Chem B. 2006;110(33):16248-1653.

8. Hindi KM, Ditto AJ, Panzner MJ, et al. The antimicrobial efficacy of sustained release silver carbene complex-loaded L-tyrosine polyphosphate nanoparticles: characterization, in vitro and in vivo studies. Biomaterials. 2009;30(22):3771-37719.

9. Paul D, Paul S, Roohpour N, Wilks M, Vadgama P. Antimicrobial, mechanical and thermal studies of silver particle-loaded polyurethane. J Funct Biomater. 2013;4(4):358-375.

10. Van der Zande M, Vandebriel RJ, Van Doren E, et al. Distribution, elimination, and toxicity of silver nanoparticles and silver ions in rats after 28-day oral exposure. ACS Nano. 2012;6(8):7427-7442.

11. Qu D, Sun W, Chen Y, Zhou J, Liu C. Synthesis and in vitro antineoplastic evaluation of silver nanoparticles mediated by Agrimoniae herba extract. Int J Nanomedicine. 2014;15(9):1871-1882.

12. Shan B, Cai YZ, Brooks JD, Corke H. The in vitro antibacterial activity of dietary spice and medicinal herb extracts. Int J Food Microbiol. 2007;117(1):112-119.

13. Song JH, Kim SK, Chang KW, Han SK, Yi HK, Jeon JG. In vitro inhibitory effects of Polygonum cupidatum on bacterial viability and virulence factors of Streptococcus mutans and Streptococcus sobrinus. Arch Oral Biol. 2006;51(12):1131-1140.

14. Oetting LL, Utiyama CE, Giani PA, et al. Effects of antimicrobials and herbal extracts on intestinal microbiology and diarrhea incidence in weanling pigs. Rev Bras Zootec. 2006;35(5):2013-2017.

15. Pedroso AA, Oetting LL, Utiyama CE, et al. Spacial variability of intestinal bacterial population of swine supplemented with antimicrobial or herbal extracts. Rev Bras Zootec. 2006;34(4):1225-1233.

16. Kokoska L, Polesny Z, Rada V, Nepovim A, Vanek T. Screening of some Siberian medicinal plants for antimicrobial activity. J Ethnopharmacol. 2002;82(1):51-53.

17. Copland A, Nahar L, Tomlinson CT, et al. Antibacterial and free radical scavenging activity of the seeds of Agrimonia eupatoria. Fitoterapia. 2003;74(1-2):133-135.

18. Lu C, Luo X, Luo R, et al. Assessment of antibacterial properties and the active ingredient of plant extracts and its effect on the performance of crucian carp (Carassius auratus gibelio var. E'erqisi, Bloch). J Sci Food Agric. 2013;93(4):902-909.

19. Zhang XQ, Gu HM, Li XZ, Zhu ZN, Chen YS, Li Y. Anti-Helicobacter pylori compounds from the ethanol extracts of Geranium wilfordii. J Ethnopharmacol. 2013;147(1):204-207.
20. Vinothkumar TS, Rubin MI, Balaji L, Kandaswamy D. In vitro evaluation of five different herbal extracts as an antimicrobial endodontic irrigant using real time quantitative polymerase chain reaction. $J$ Conserv Dent. 2013;16(2):167-170.

21. Niraimathi KL, Sudha V, Lavanya R, Brindha P. Biosynthesis of silver nanoparticles using Alternanthera sessilis (Linn.) extract and their antimicrobial, antioxidant activities. Colloids Surf B Biointerfaces. 2013;102:288-291.

22. Link S, Wang ZL, El-Sayed MA. Alloy formation of gold-silver nanoparticles and the dependence of the plasmon absorption on their composition. J Phys Chem B. 1999;103(18):3529-3533.

23. Martinez-Gutierrez F, Olive PL, Banuelos A, et al. Synthesis, characterization, and evaluation of antimicrobial and cytotoxic effect of silver and titanium nanoparticles. Nanomedicine. 2010;6(5):681-688.

24. Burkowska-But A, Sionkowski G, Walczak M, et al. Influence of stabilizers on the antimicrobial properties of silver nanoparticles introduced into natural water. J Environ Sci (China). 2014;26(3):542-549.

25. Parashar UK, Kumar V, Bera T, Saxena PS, et al. Study of mechanism of enhanced antibacterial activity by green synthesis of silver nanoparticles. Nanotechnology. 2011;22(41):415104.

26. Sun W, Chen Y, Gao X, Liu CY, Qu D. [Preparation and characterization of Sanguisorbae Radix/nano-silver composites]. Zhong Cao Yao. 2013;44(24):3465-3470. Chinese.

27. Loo YY, Chieng BW, Nishibuchi M, Radu S. Synthesis of silver nanoparticles by using tea leaf extract from Camellia sinensis. Int $J$ Nanomedicine. 2012;7:4263-4267.

28. Kalishwaralal K, Banumathi E, Ram Kumar Pandian S, et al. Silver nanoparticles inhibit VEGF induced cell proliferation and migration in bovine retinal endothelial cells. Colloids Surf B Biointerfaces. 2009; 73(1):51-57.

29. Kumar V, Yadav SK. Plant-mediated synthesis of silver and gold nanoparticles and their applications. J Chem Technol Biotechnol. 2009; 84:151-157.

30. Cruz D, Falé PL, Mourato A, Serrelheiro ML, Lino AR. Preparation and physicochemical characterization of Ag nanoparticles biosynthesized by Lippia citriodora (Lemon Verbena). Colloids Surf B Biointerfaces. 2010;81(1):67-73.

31. Daniel MC, Astruc D. Gold nanoparticles: assembly, supramolecular chemistry, quantum-size related properties, and applications toward biology, catalysis, and nanotechnology. Chem Rev. 2004;104(1): 293-346.

32. Bouwmeester H, Poortman J, Peters RJ, et al. Characterization of translocation of silver nanoparticles and effects on whole-genome gene expression using an in vitro intestinal epithelium coculture model. ACS Nano. 2011;5(5):4091-4103.

33. Borchert H, Shevchenko EV, Robert A, et al. Determination of nanocrystal sizes: a comparison of TEM, SAXS and XRD studies of highly monodisperse CoPt3 particles. Langmuir. 2005;21(5): 1931-1936.

34. Elavazhagan T, Arunachalam KD. Memecylon edule leaf extract mediated green synthesis of silver and gold nanoparticles. Int J Nanomedicine. 2011;6:1265-1278.

35. Shanmugasundaram T, Radhakrishnan M, Gopikrishnan V, Pazhanimurugan R, Balagurunathan R. A study of the bactericidal, antibiofouling, cytotoxic and antioxidant properties of action bacterially synthesized silver nanoparticles. Colloids Surf B Biointerfaces. 2013;111: 680-687.

36. Chandran SP, Chaudhary M, Pasricha R, et al. Synthesis of gold nanoparticles and silver nanoparticles using Aloe vera plant extract. Biotechnol Prog. 2006;22(2):577-583.

37. Quiros I, Yamada M, Kubo K, Mizutani J, Kurihara M, Nishihara H. Preparation of alkanethiolate-protected palladium nanoparticles and their size dependence on synthetic conditions. Langmuir. 2002; 18(4):1413-1418 
38. Neu HC. The crisis in antibiotic resistance. Science. 1992;257(5073): 1064-1073.

39. Craig WA. Antimicrobial resistance issues of the future. Diagn Microbiol Infect Dis. 1996;25(4):213-217.
40. Zhang Y, Chen F, Zhuang J, et al. Synthesis of silver nanoparticles via electrochemical reduction on compact zeolite film modified electrodes. Chem Commun (Camb). 2002;23:2814-2815. 


\section{Supplementary materials}

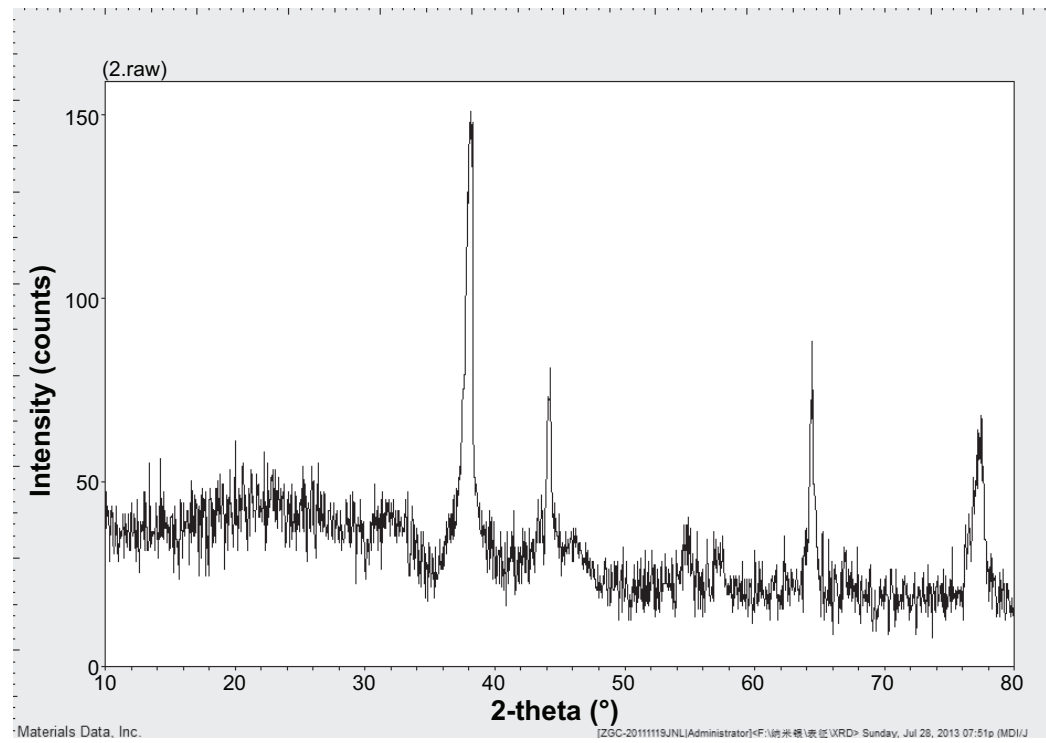

Figure SI X-ray diffraction patterns for Polygonum cuspidatum Sieb. et-coated silver nanoparticles.

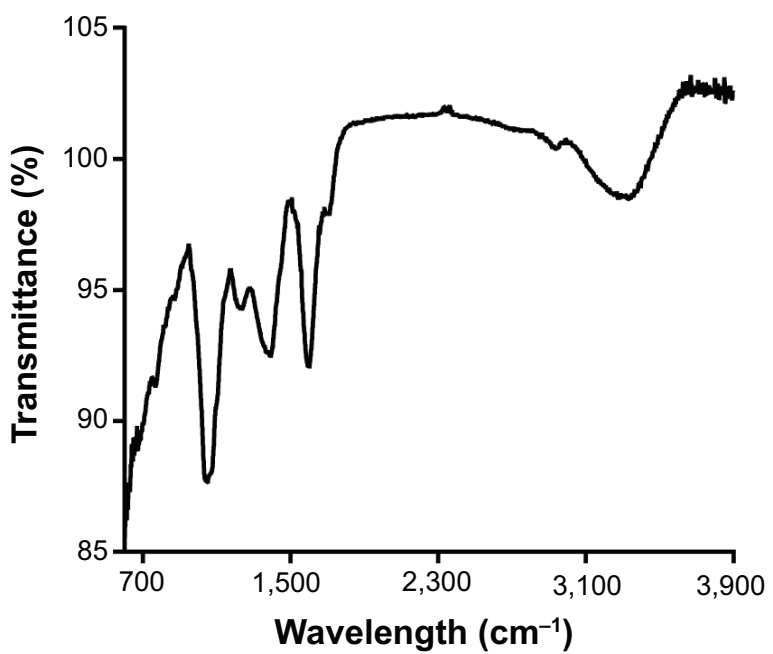

Figure S2 Fourier transform infrared absorption spectra for Polygonum cuspidatum Sieb. et-coated silver nanoparticles.

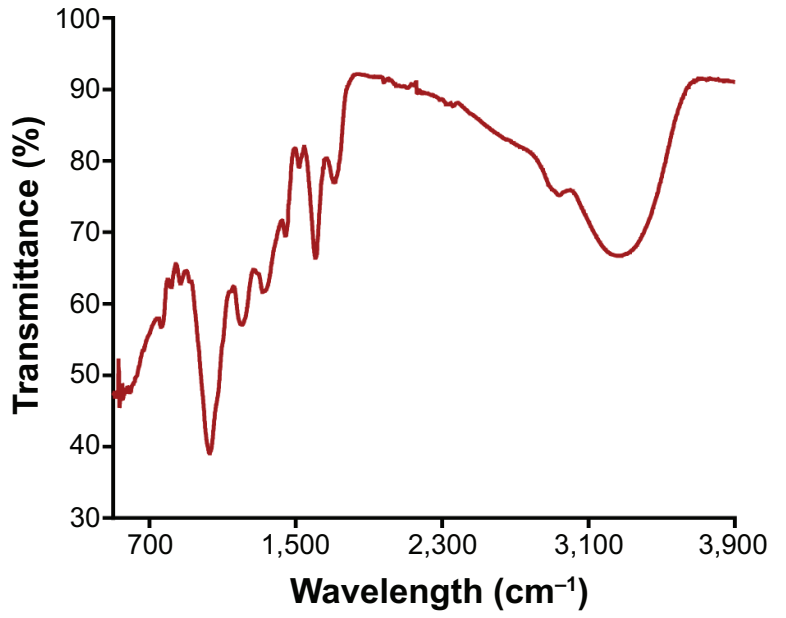

Figure S3 Fourier transform infrared absorption spectra of Polygonum cuspidatum Sieb. et. extraction.

\section{Publish your work in this journal}

The International Journal of Nanomedicine is an international, peerreviewed journal focusing on the application of nanotechnology in diagnostics, therapeutics, and drug delivery systems throughout the biomedical field. This journal is indexed on PubMed Central, MedLine, CAS, SciSearch $\AA$, Current Contents $\AA /$ Clinical Medicine,
Journal Citation Reports/Science Edition, EMBase, Scopus and the Elsevier Bibliographic databases. The manuscript management system is completely online and includes a very quick and fair peer-review system, which is all easy to use. Visit http://www.dovepress.com/ testimonials.php to read real quotes from published authors. 